\title{
SFHs Across the Merging Disks of Arp 244 - from FUV to MIR
}

\author{
Hong-Xin Zhang ${ }^{1,2}$, Yu Gao ${ }^{1}$ and Xu Kong ${ }^{3}$ \\ ${ }^{1}$ Purple Mnt. Obs, Chinese Academy of Sciences, 2 West Beijing Road, Nanjing 210008, China \\ ${ }^{2}$ Graduate School, Chinese Academy of Sciences, Beijing 100039, China \\ ${ }^{3}$ Center for Astrophysics, University of Science and Technology of China, Hefei 230026, China
}

\begin{abstract}
Including MIPS $24 \mu \mathrm{m}$ dust emission in the multi-band stellar population analysis, we roughly constrain the star formation histories (SFHs) of MIPS $24 \mu \mathrm{m}$ selected star-forming regions across the merging disks of the Antennae galaxies. While the overlap regions hold the highest ratios of young over intermediate populations, the western-loop regions have the highest ratios of intermediate to old populations. We find two sequential star formation (SF) paths in the overlap regions, which we interpret as the imprint of the interpenetrating process of the two merging disks following their second close encounter.
\end{abstract}

Keywords. galaxies: the "Anennae" galaxies-galaxies: interactions- galaxies: stellar content

\section{Introduction}

The Antennae is the nearest prototypal major merger between two gas-rich spiral galaxies, which provides us with a unique opportunity to study the merger-induced SF process as a consquence of galaxy interaction in detail. In this study, with the high resolution Spitzer IR dust emission data being included into population analysis, we show that the degeneracy between population and extinction is broken to a great extent. Thus, we can roughly constrain the SFHs within the Antennae using spectral energy distributions (SEDs) from FUV to IR. We here present the broadband SEDs of starforming regions selected mainly from the $24 \mu \mathrm{m}$ image and briefly describe our method to constrain the SFHs across the merging disks. Finally we point out two sequential SF paths across the overlap regions.

\section{Data and Methodology}

The archival data used in this analysis are as follows, FUV and NUV images from GALEX, Four broad band (F336W, F439W, F555W, F814W) and one narrow band $(\mathrm{F} 658 \mathrm{~N} ; \mathrm{H} \alpha)$ images from WFPC2 aboard the HST. JHKs imagery from 2MASS. IRAC 3.6, 4.5, 5.8, 8.0 $\mu \mathrm{m}$ and MIPS $24 \mu \mathrm{m}$ images from Spitzer. Prior to extracting our multiwavelength photometry, all images were background removed, aligned, resolutionmatched and resampled to the same pixel scale $\left(1.5^{\prime \prime}\right)$. Then we perform photometry for 34 positions of each $9^{\prime \prime}(\sim 800 \mathrm{pc})$ diameter circular aperture (Fig. 1). These are mainly the emission peaks in MIPS $24 \mu \mathrm{m}$ map corresponding to the intense star-forming regions selected from $24 \mu \mathrm{m}$ image. We fit the SEDs (FUV-Ks) with superpositions of three (<10Myr, 10Myr-300Myr, >300Myr) single stellar populations (SSPs) models from STARBURST99. We adopt the Charlot \& Fall (2000) extinction recipe. With the combination of $24 \mu \mathrm{m}$ and $\mathrm{H} \alpha$ (Calzetti et al. 2007), we fix the extinction of young $(<10 \mathrm{Myr})$ populations. Then, the population-extinction degeneracy is broken largely, thus reliable mass fraction ratios between different populations can be obtained. 


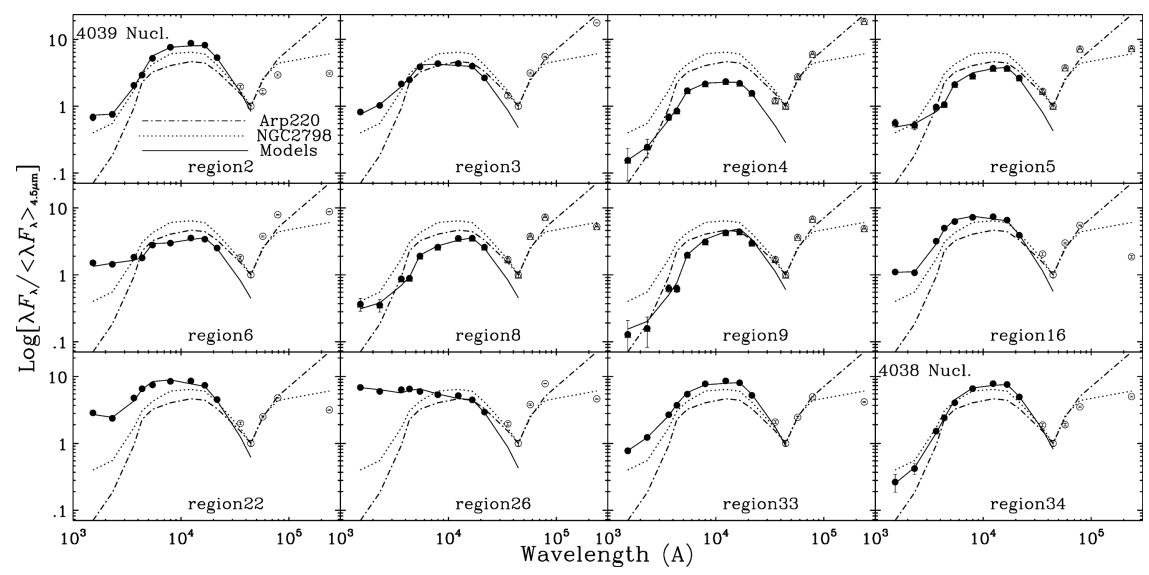

Figure 2. Display of FUV-24 $\mu \mathrm{m}$ SEDs of some representative regions marked in Figure 1. Also plotted for comparison are Arp220 and local HII galaxy NGC2798. The best-fit broadband SEDs of the composite population models are plotted as solid lines.

\section{Results and Discussion}

SEDs of some representative SF regions are shown in Fig. 2. Obviously, the overlap regions have generally higher ratios of $24 / 8 \mu \mathrm{m}$ than all the other regions, indicating currently there are the most intense SF sites. On the contrary, the western-loop regions have the strongest UV emission and largest UV excesses, yet low $24 / 8 \mu \mathrm{m}$ ratios, implying the later SF stage of the recent violent SF episode.

In general, our population analysis is consistent with the SF scenario that rather extended moderate SF coexists with the confined, intense starbursts in the overlap and western - loop regions. The overlap regions, which generally have the highest fractions of young populations, are experiencing much more violent SF than all the other regions. One interesting finding is that, across the overlap regions, the north edge (i.e. region 9) and the south edge (i.e. region 4 ) have the significantly higher mass ratios of young to intermediate populations than the central regions. We would like to interpret this as two sequential SF paths following the pair's second close encounter. Such SF sequences could be naturally explained with the physical mechanism proposed by Jog \& Solomon (1992).

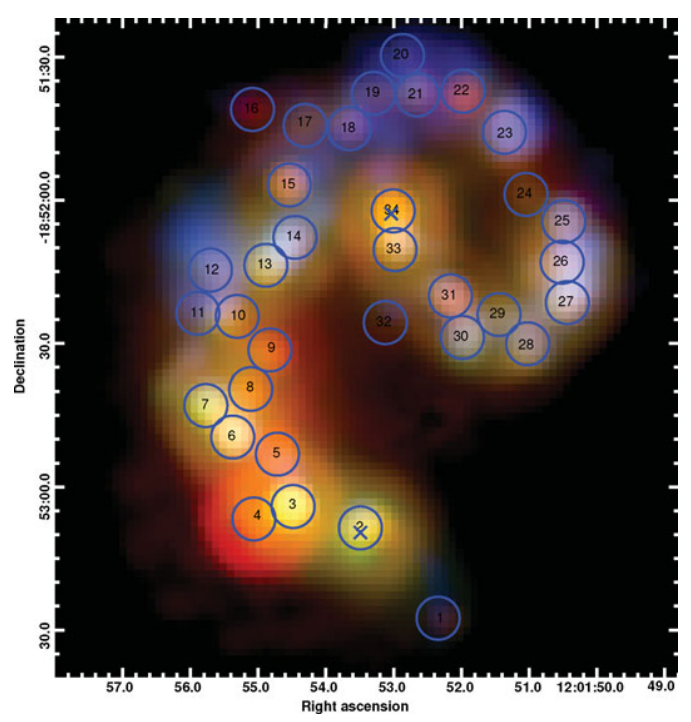

Figure 1. Circular apertures of $9^{\prime \prime}$ diameter selected mainly as $24 \mu \mathrm{m}$ peaks are superposed on threecolor composites generated from MIPS $24 \mu \mathrm{m}($ red), degraded HST $\mathrm{H} \alpha$ (green), and GALEX FUV(blue) maps of the Antennae.

\section{References}

Calzetti, D., Kennicutt, R. C., Engelbracht, C. W. et al. 2007, ApJ, 666, 870

Charlot, S. \& Fall, S. M. 2000, ApJ, 539, 718

Jog, C. J. \& Solomon, P. M. 1992, ApJ, 387, 152 